\title{
Using IoT to Improve Uptime and Customer Satisfaction
}

\author{
Marc Schweitzer \\ Carl Zeiss X-ray Microscopy, Inc., Pleasanton, California, United States
}

\begin{abstract}
One of the constant challenges facing managers in central microscopy facilities is the uptime and maintenance of their instruments. Downtime due to performance issues or repairs has a direct negative impact on the operation of the facility, from both a scientific and financial perspective. As vendors, we must commit ourselves to finding new, innovative, and effective ways to mitigate these issues and improve the user experience. This talk will describe one such method: an IoT (Internet of Things) system called Predictive Service used to connect to and monitor the condition of installed microscope instruments. ZEISS has deployed this system to most of their microscope modalities including Light Microscopy (LM), Electron Microscopy (EM) and X-ray Microscopy (XRM). At present, there are over 780 instruments listed worldwide.[i] Implementation of this system has revealed new opportunities and challenges for the Service organization which are being systematically addressed. Although objective data is still being collected, initial reports are promising and show a steady decrease in Mean Time To Repair (MTTR), increased uptime and improved customer satisfaction.
\end{abstract}

\section{Introduction}

As the installed base of microscopes continues to grow, and new systems increase in complexity, vendor service organizations must look for scalable process improvements that leverage the instrument technology to meet customer's expectations. At ZEISS, the historical process is that the customer must call the Customer Service Center after degraded performance is observed. Once the case is logged, a service engineer is dispatched to the customer to diagnose and correct the problem. If necessary, the customer engineer will send system log files and other information to the Technical Support Group (TSG) at the product center for further analysis via email. A further escalation to the product group R\&D system engineers may also be necessary. Once diagnosed, a Plan of Action (POA) is developed and sent to the customer engineer on-site to implement. If a spare part is required, then it is ordered but requires a subsequent visit by the customer engineer to install. Meanwhile, the customer must wait with their system in a degraded state for the process to complete. Often the downtime is dominated by delays in diagnosis and/or spare part delivery times.

\section{ZEISS XRM Example}

Many modern sophisticated microscopes use PCs for control and/or data acquisition. Thus, much of the instrument functionality is already monitored by the instrument PC and available to assist Customer Engineers for diagnostic purposes when they arrive onsite. Years ago, ZEISS XRM Technical Support Group started using commercially available PC to PC portals to allow service personnel to connect remotely to the instrument PCs. This program worked very well and allowed routine problems to be addressed without requiring the service personnel to be on-site. However, the customer still had to initiate the service request and then initiate the PC connection after the service engineer makes contact.

A better way was sought to establish a permanent connection between the customer's instrument PC and a secure server using an IoT service which allows critical system performance data such as error logs to 
be reviewed remotely without burdening the customer. This way, ZEISS Customer Engineers could monitor the system when convenient and proactively intervene before the customer noticed degraded performance.

\section{Implementation}

To implement an IoT system, the PTC Thingworx platform was chosen because it had several out-of-thebox features that would offer usable functionality quickly. Together with in-house developers, ZEISS partnered with an external developer, with previous experience using Thingworx.[ii] The prebuilt platform and consultant accelerated development to the point where it enabled the IoT service to be deployed in a cost-effective manner in under 1 year. By the end of the following year over 500 systems were signed up. The latest implementation allows ZEISS to "push" updates to the Agents on the Instrument PCs at the end of each sprint permitting very rapid bug fixing and new feature deployment.

\section{IoT enabled Improved Service Model.}

Having off-site access to the system performance data is a substantial advantage to the field service personnel. But to even further leverage this data, especially during the early days of this new program, ZEISS XRM is piloting a new system in which a small cross functional team from the Product Center including System, Software and Technical Support Engineers hold a daily standup meeting of about 15 minutes to discuss the health of connected systems. During these meetings any systems that have shown a performance issue in the previous day are discussed and analyzed. A Plan of Action (POA) along with the level of urgency is documented for each system and communicated to each ZEISS Customer Engineer via email for follow-up.

\section{Results}

Since the daily standups started, most of the identified issues were corrected either remotely or on-site by the ZEISS Customer Engineer before the customer noticed degraded performance. The ability to have an instrument repaired before it actually breaks is of course a major benefit to the user and represents a substantial breakthrough in how we are able to proactively support our customers. In a couple of cases, the team anticipated a component failure in the future and instructed the Customer Engineer to procure the component in advance so it could be carried on-site when failure was finally confirmed. These actions combined with better customer engineer training and logistical improvements have led to a steady decline in MTTR (See Figure 1) for the last year with a corresponding increase in system uptime and customer satisfaction. 


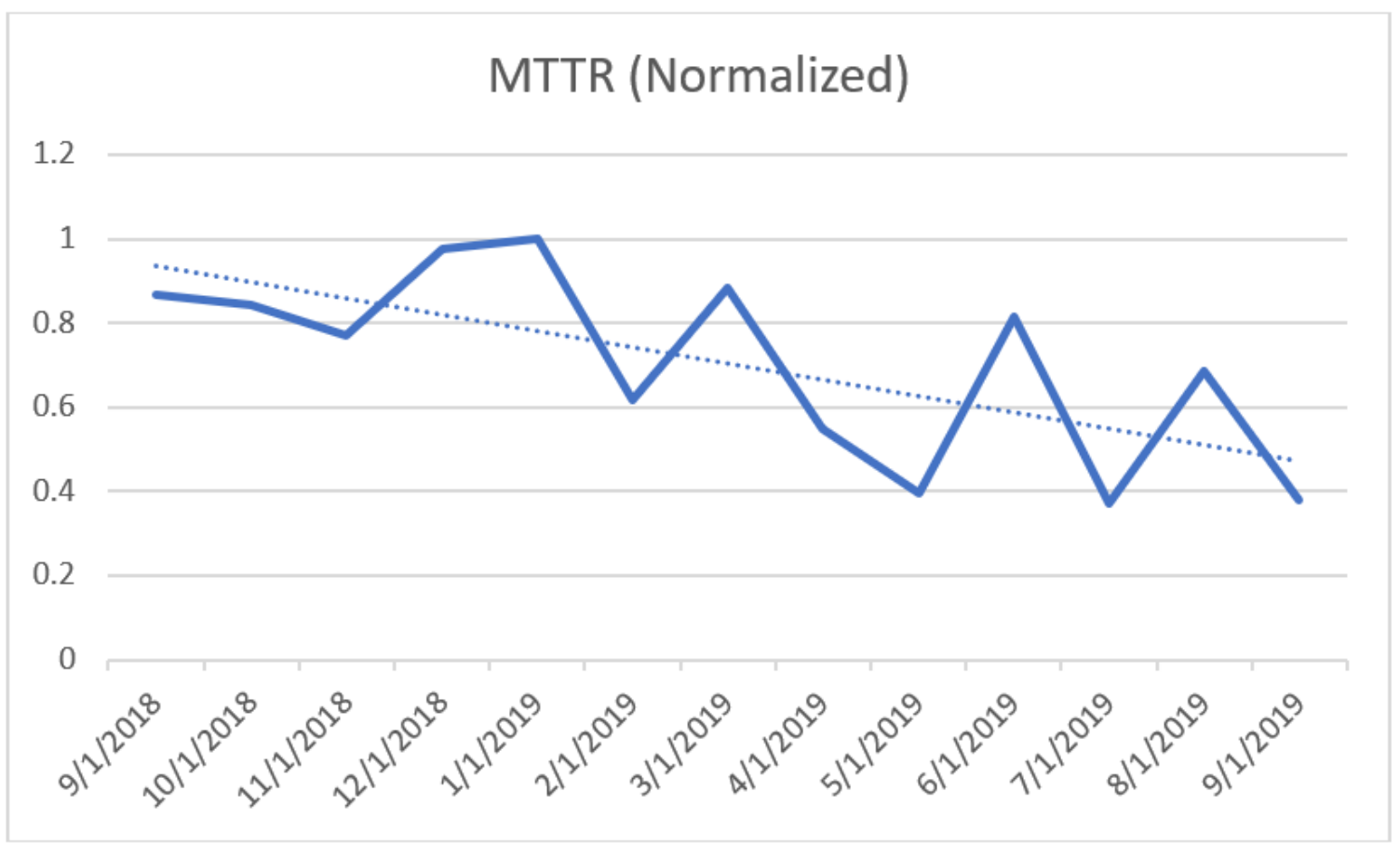

Figure 1. Normalized Mean Time To Repair (MTTR) for ZEISS XRM Systems.

\section{References}

[1] Predictive Service "Active" system count as of 2/20/20.

[1] DoubleSlash Net-Business Gmbh 